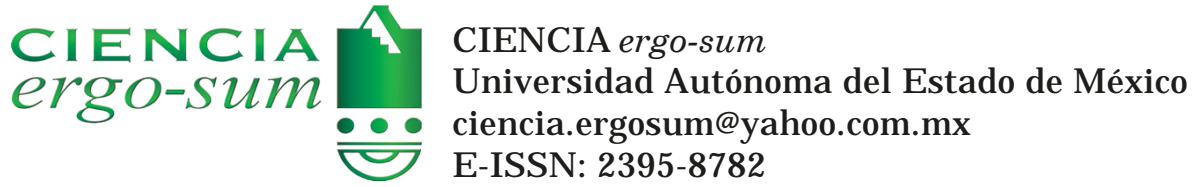

\title{
Agroquímicos organofosforados y su potencial daño en la salud de trabajadores agrícolas del campo sonorense
}

Alvarado I barra, J uana; Valencia López, Carlos Armando; Castillo Moreno, Manuel Rogelio; Luna Reyes, Pedro

David; Borboa Servin, J uan Andrés; Mexia Apodaca, Martín Eduardo y Ruiz Sandoval, Néstor Cipriano Agroquímicos organofosforados y su potencial daño en la salud de trabajadores agrícolas del campo sonorense CIENCIA ergo-sum, vol. 26, núm. 1, marzo-junio 2019| e43

Universidad Autónoma del Estado de México, México

Esta obra está bajo una Licencia Creative Commons Atribución-NoComercial-SinDerivar 4.0 Internacional.

Alvarado I barra, J ., Valencia López, C. A., Castillo Moreno, M. R., Luna Reyes, P. D., Borboa Servin, J . A., Mexia A podaca, M. E. y Ruiz Sandoval, N. C. (2018). Agroquímicos organofosforados y su potencial daño en la salud de trabajadores agrícolas del campo sonorense. CIE NCIA ergo-sum, 26(1). https://doi.org/10.30878/ces.v26nla8 


\title{
Agroquímicos organofosforados y su potencial daño en la salud de trabajadores agrícolas del campo sonorense
}

Organophosphate agrochemicals and its potential damage in the health of agricultural workers of Sonora, Mexico

Juana Alvarado Ibarra

Universidad de Sonora, México

juana.alvarado@unison.mx

Recepción: 10 de agosto de 2017

Carlos Armando Valencia López

Universidad de Sonora, México

car_16_98@hotmail.com

Manuel Rogelio Castillo Moreno

Universidad de Sonora, México

maropo3@hotmail.com

Pedro David Luna Reyes

Universidad de Sonora, México

lunareyespe89@gmail.com

Juan Andrés Borboa Servin

Universidad de Sonora, México

juan_andres354@hotmail.com

Martín Eduardo Mexia Apodaca

Universidad de Sonora, México

martin_mexia05@hotmail.com

Néstor Cipriano Ruiz Sandoval

Universidad de Sonora, México

nestor_ruiz27@outlook.com

\section{Resumen}

El uso de agroquímicos es necesario para mantener el rendimiento promedio de los cultivos. Sin embargo, los efectos causados en los trabajadores agrícolas representan un problema de salud. La determinación de la actividad de la colinesterasa en la sangre es un valioso indicador de exposición a agroquímicos organofosforados. Con base en lo anterior, se explora la posibilidad de intoxicaciones agudas grado 1 y se determinan los valores de acetilcolinesterasa en 25 personas que laboran en actividades agrícolas y en 5 como muestra control. De la población estudiada, $86 \%$ presentó valores normales de la actividad de la colinesterasa. No obstante, los resultados sirven como indicadores biológicos de intoxicación y son importantes para el seguimiento y detección de los efectos tóxicos producidos.

Palabras Claves: acetilcolinesterasa, agroquímicos organofosforados, intoxicación, campo sonorense.

\begin{abstract}
The agrochemicals are necessary to maintain the crop production in yields. However, the effects caused on agricultural workers by exposure represent a health problem, so, the determination of blood cholinesterase activity is a valuable indicator of exposure to them. In this study we explored the possibility of acute poisoning grade 1 and the values of acetylcholinesterase that were determined for 25 people who work in agricultural activities and 5 were taken as a control sample. Although the 86 percent of the study population had normal values of cholinesterase activity, the results should be used as biological intoxication indicators and they are an important part of the monitoring and detection of toxic effects produced by exposure to organophosphorus agrochemicals.
\end{abstract}

KEYWORDS: acetylcholinesterase, organophosphate agrochemicals, intoxication, Sonora crop yield. 


\section{INTRODUCCIÓN}

México cuenta con una gran diversidad de climas y tipos de suelos en los que se puede sembrar una vasta variedad de cultivos. La agricultura es sin duda una de las actividades primarias más importantes del país, ya que cuenta con una superficie sembrada de 21938183.83 ha (SIAP, 2016). De las unidades de producción, se estima que un $94 \%$ está destinado para el autoconsumo y el mercado local y $6 \%$ produce para el mercado nacional e internacional (Mota, 2012). El estado de Sonora tiene una superficie sembrada de 662595.60 ha, el equivalente a 3.02\% de la superficie que se cultiva en el país, colocándose en el décimo sexto lugar de la producción agrícola nacional (SIAP, 2016). Por lo anterior y por la posición geográfica del estado, cercano al mercado estadounidense, el campo sonorense es atractivo para la población de otras partes del país, sobre todo de indígenas del sureste de México, quienes llegan a emplearse como jornaleros en el sector agrícola; se calcula que anualmente llega un aproximado de 60000 personas a los campos del cultivo de vid (Notimex, 2017), pepino y calabaza (Comunica Campeche, 2016).

A fin de tener una mayor productividad agrícola y reducir pérdidas por plagas durante el proceso de cultivo en México, aun cuando existan alternativas de uso de agroquímicos con perspectiva ecológica y sostenible (Pérez et al., 2010), se tiene todavía la práctica de utilizar agroquímicos y productos fitosanitarios con elevada toxicidad para los humanos, animales y algunas plagas (Arellano-Aguilar y Rendón, 2016). Aunado a lo anterior, en los campos agrícolas mexicanos se usan agroquímicos que están prohibidos en otros países (Tourliere, 2015; Arellano-Aguilar y Rendón, 2016), cuyos componentes químicos pueden ingresar a los organismos por inhalación de vapores o de polvos, por absorción gastrointestinal y por penetración a través de la piel o de las mucosas, los cuales tienen acción insecticida, fungicida, herbicida, acaricida, nematicida, molusquicida, avicida o rodenticida (Milla-Cotos y Palomino-Horna, 2002).

Sonora se encuentra dentro de los estados con mayor uso de agroquímicos (Albert, 2015), por lo que participa fuertemente con un aproximado de 110873 toneladas de ingredientes activos que se consumen por año en el país (FAOSTAT, 2017). Uno de los agroquímicos que más se usa en los campos mexicanos es el de los compuestos organofosforados (OF's) (Arellano-Aguilar y Rendón, 2016). En el caso de Sonora se han usado en los cultivos de hortalizas, frutales, granos, algodón e inclusive en los almacenes de granos (Silveira et al., 2011).

Los compuestos organofosforados tienen sustancias que persisten en el ambiente poco tiempo (GutiérrezCoronado et al., 2012; Pérez-Herrera et al., 2012); no obstante, sus metabolitos o sus productos de degradación son considerados contaminantes, ya que al entrar al ambiente afectan tanto a organismos benéficos como al humano (Badii y Varela, 2008). Aunque la mayoría de las personas tiene historial de exposición a los agroquímicos organofosforados, los trabajadores agrícolas lo están al doble por residir cerca de las áreas de cultivo y también tienden a presentan alto riesgo de toxicidad aguda y crónica. Entre los efectos que muestran están los neurofisiológicos, endócrinos, inmunológicos, además de algunos tipos de cánceres, cardiopatías y diabetes (Alavanja et al., 2004; Palacios-Nava y Moreno-Tetlacuilo, 2004).

La exposición laboral crónica a organismos organofosforados conlleva a evaluar la toxicidad por medio de monitorización biológica que, según la Comisión Intersecretarial para el Control del Proceso y uso de Plaguicidas, Fertilizantes y Sustancias Tóxicas (CICOPLAFEST, 1991), debe incluir, entre otros, la determinación de la acetilcolinesterasa en sangre, ya que representa el blanco molecular de la toxicidad de estos compuestos químicos. Aunque con frecuencia los organismos organofosforados ejercen su efecto inhibitorio sobre la acetilcolinesterasa en las terminaciones nerviosas, clínicamente se monitorea la colinesterasa plasmática o sérica debido a que su concentración es mucho mayor y los cambios en sus niveles son detectables con facilidad por las técnicas de laboratorio (Shelat, 2017).

Existen varios trabajos donde se han evaluado parámetros hematológicos y de otros fluidos corporales de individuos expuestos a organismos organofosforados, principalmente a personal ocupado en labores de campo (Badii y Varela, 2008; Pérez et al., 2010; Camarena-Ojinaga et al., 2012; Gutiérrez-Coronado et al., 2012), ya 
que por cuestiones laborales y ambientales son un grupo vulnerable por su constante exposición a agroquímicos, (Seefoó-Lujan, 2006; Pérez et al., 2010). En México el manejo de agroquímicos por lo general se realiza en un contexto de falta de asesoría técnica y de medidas de protección insuficientes, inadecuadas o en muchas ocasiones nulas (Pérez-Herrera et al., 2012), y así lo demuestra el Informe Anual 2016 de la Protección de Cultivos, Ciencia y Tecnología, A. C. (PROCCYT, 2016), que indica que en el año reportado hubo afectaciones por agroquímicos tanto a personal ocupacional como a personal no ocupado y a animales debido al mal manejo y almacenaje de productos, a no utilizar equipo de protección personal y por encontrarse en las cercanías de las zonas donde se aplica.

Desde el punto de vista de leyes que rigen la protección de la salud y el ambiente por el desarrollo de actividades agrícolas, México cuenta con el Proyecto de Norma Oficial Mexicana PROY-NOM -003-STPS-2016, en el que se indica que es aplicable en todos los centros de trabajo donde se realicen actividades agrícolas que van desde la preparación del terreno hasta la cosecha y en él también se establecen las disposiciones que deberán adaptarse a los centros de trabajo, a efecto de prevenir los riesgos a los que están expuestos los trabajadores que desarrollen actividades agrícolas (Segob, 2017).

Es sabido que el grado de intoxicación depende de factores como la fórmula del agroquímico, el ingrediente activo, el tiempo de exposición, el contacto directo o indirecto, la cantidad empleada, las mezclas de sustancias, el clima y temporada del año, así como la edad y el género de las personas (Dosemeci et al., 2002; Rothlein et al., 2006). Por estos antecedentes, este trabajo explora el potencial daño en la salud de un grupo de hombres agricultores del municipio de Bacerac y en un grupo de mujeres recolectoras de uva de mesa de un campo agrícola de la Costa de Hermosillo, ambos en el estado de Sonora, México, a través de la aplicación de un cuestionario exploratorio de prácticas laborales y de determinar la actividad de la colinesterasa sérica como indicador de exposición a agroquímicos organofosforados.

\section{Materiales y métodos}

\section{1. Sitios de estudio}

La investigación se llevó a cabo en dos municipios del estado de Sonora (identificados en el mapa 1). Uno se realizó con agricultores del municipio de Bacerac, ubicado en el noreste del estado, en las coordenadas geográficas $30^{\circ} 15^{\prime} 51^{\prime \prime} \mathrm{N}$ y $108^{\circ} 40^{\prime} 00^{\prime \prime} \mathrm{O}$, con elevación media de 1370 msnm (INEGI, 2010). De la práctica agrícola del municipio, $70 \%$ es de apoyo al sector pecuario y $30 \%$ se destina al cultivo de granos básicos como frijol, maíz y trigo. Otros de los cultivos son avena, cebada y alfalfa (SIAP, 2014).

El otro estudio fue en el campo agrícola ubicado en la calle 36 Norte, con coordenadas geográficas $29^{\circ} 07^{\prime}$ 34 ” N y $111^{\circ} 43^{\prime} 28^{\prime \prime}$ O, perteneciente a la comisaría Miguel Alemán de la Costa de Hermosillo. La calle 36 Norte se encuentra al poniente de Hermosillo, en el kilómetro 87 al norte de la carretera a Bahía de Kino, el cual colinda con la carretera a Puerto Libertad (Cisneros-González, 1990). Presenta un clima semidesértico, con temperatura media anual de $24^{\circ} \mathrm{C}$ que oscila entre $-3{ }^{\circ} \mathrm{C}$ como mínima y $46{ }^{\circ} \mathrm{C}$ como máxima, la precipitación media anual es de $200 \mathrm{~mm}$ y una tasa de evapotranspiración de hasta $2560 \mathrm{~mm}$ (Salazar-Adams et al., 2012). Una de las actividades económicas primordiales de la zona es la producción hortofrutícola para el mercado internacional, por lo que es indispensable cumplir con los estándares de inocuidad alimentaria y de normas de protección humana (Gutiérrez-Coronado et al., 2012).

\section{ObTención de MUestras}

El diseño metodológico fue descriptivo, transversal y aleatorio entre las personas que aceptaron participar. Los municipios seleccionados fueron visitados en abril de 2017. En Bacerac participaron 12 agricultores nativos y en 
la Costa de Hermosillo participaron 13 mujeres procedentes del estado de Guerrero que se desempeñan como recolectoras de uva de mesa.

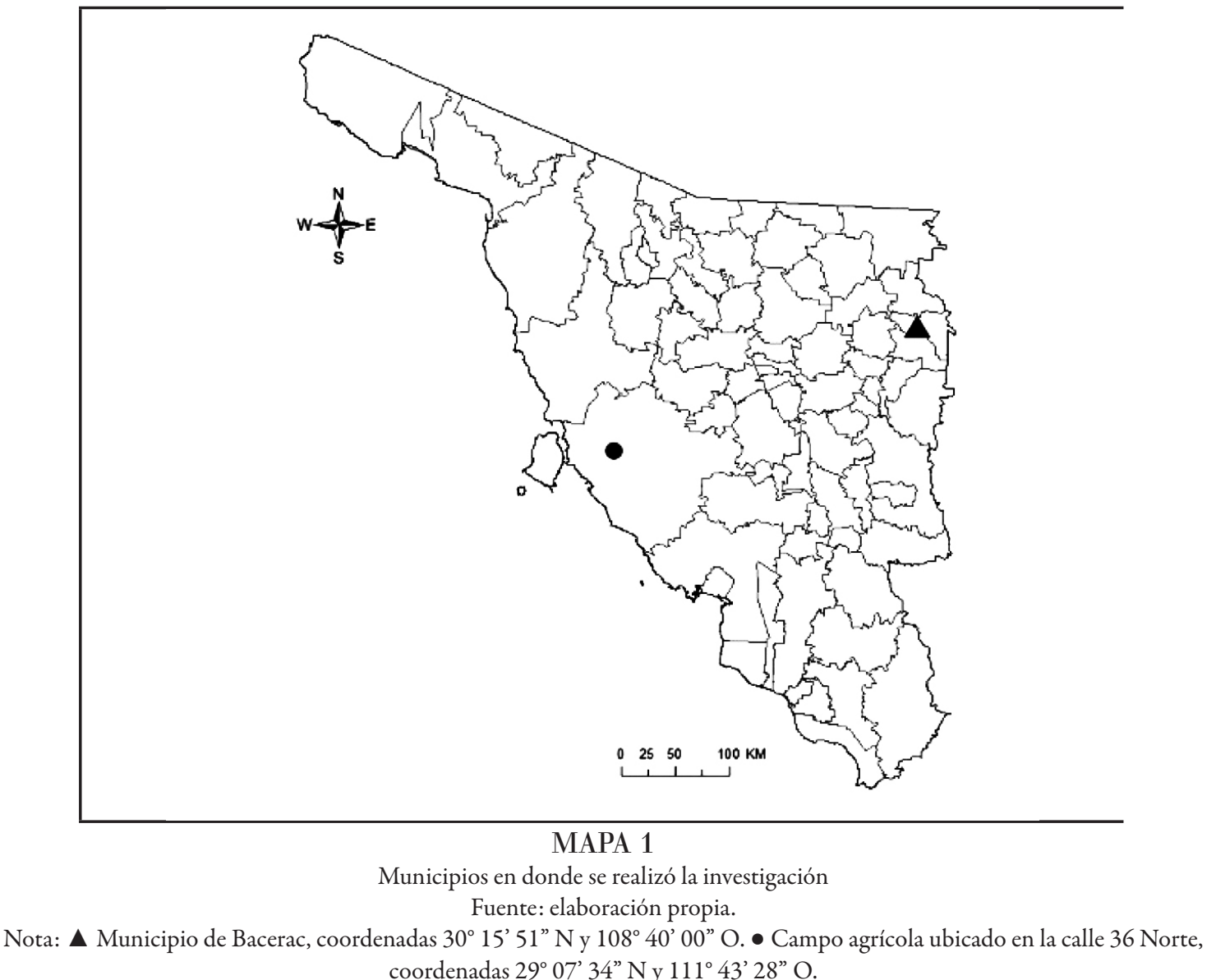
coordenadas $29^{\circ} 07^{\prime} 34^{\prime \prime} \mathrm{N}$ y $111^{\circ} 43^{\prime} 28^{\prime \prime} \mathrm{O}$.

A los participantes se les aplicó un cuestionario para recabar datos de sus prácticas laborales, enfermedades referidas y síntomas persistentes. Respecto a la intoxicación aguda por organofosforados, se preguntó acerca de sintomatología sufrida durante los últimos dos meses de trabajo, la cual se consideró como una intoxicación grado 1 o leve (Pose et al., 2000). Se les pidió la firma de un formato de consentimiento de participación voluntaria en el estudio. Posteriormente, se les tomó una muestra de sangre venosa siguiendo la metodología descrita por Gutiérrez-Coronado et al. (2012). Esta muestra fue analizada por el método colorimétrico para el parámetro de colinesterasa en un laboratorio de análisis clínicos de Hermosillo, ya que tiene estandarizado el método, está registrado ante la Secretaría de Salud en México con el número 445/04 y el químico responsable cuenta con la cédula profesional 4214232.

Se determinó la concentración de colinesterasa a través de la acetilcolinesterasa (AChE). La técnica consiste en la hidrólisis del sustrato acetiltiocolina por la enzima colinesterasa. La tiocolina liberada reacciona con un cromóforo, el ácido 5, 5 -ditiobis-2-nitrobenzoico, dando lugar como producto de reacción al ácido 5-tio-2nitrobenzoico, compuesto de color amarillo cuyo máximo de absorbancia se encuentra entre 405 y $420 \mathrm{~nm}$ de longitud de onda (Tecles y Cerón, 2003).

Se seleccionó la AChE por ser considerada la más representativa de la enzima del tejido nervioso y por su importancia en los sistemas de vigilancia al determinar mejor la toxicidad crónica (Jiménez-Diaz y SchosinskyNevermann, 2000). Esta enzima se midió en 30 individuos, conformados por 12 hombres del municipio de Bacerac, 13 mujeres de la Costa de Hermosillo y para muestra control se contó con 5 personas no relacionadas con actividades agrícolas. Aunque el valor de colinesterasa depende de variabilidad biológica interindividual e 
intraindividual, ya que se relaciona con la edad, el sexo, la grasa corporal y el embarazo (Dosemeci et al., 2002; Rothlein et al., 2006), los intervalos de referencia considerados son 4620-1 1500 UI/L para hombres y 3 930-10 $800 \mathrm{UI} / \mathrm{L}$ para mujer (Centralab, 2016).

\section{Resultados y discusión}

En la tabla 1 se presenta un concentrado de los agroquímicos organofosforados encontrados en los sitios de estudio. En el municipio de Bacerac se cultiva alfalfa y se usa como agroquímico el Metamidofos 600, comprado y aplicado por los propios agricultores. El producto, según la COFEPRIS (Gowan Mexicana, 2013, se encuentra en la categoría de altamente tóxico de acuerdo con la NOM-232-SSA1-2009 en la categoría toxicológica 2 (DOF-SSA, 2010), y en países desarrollados está prohibido su uso debido a su alta toxicidad para los organismos (Pérez-Herrera et al., 2012). Lo anterior es un problema grave, puesto que es evidente que no existe vigilancia estricta de las leyes y reglamentos de protección ambiental, aunado a la falta de asesoría técnica para el control y evaluación de las aplicaciones que en ocasiones se realizan con limitantes técnicos impactando en el ecosistema y en la salud de quienes laboran en el campo (Badii y Varela, 2008).

TABLA 1

Agroquímicos comúnmente usados en las zonas de estudio

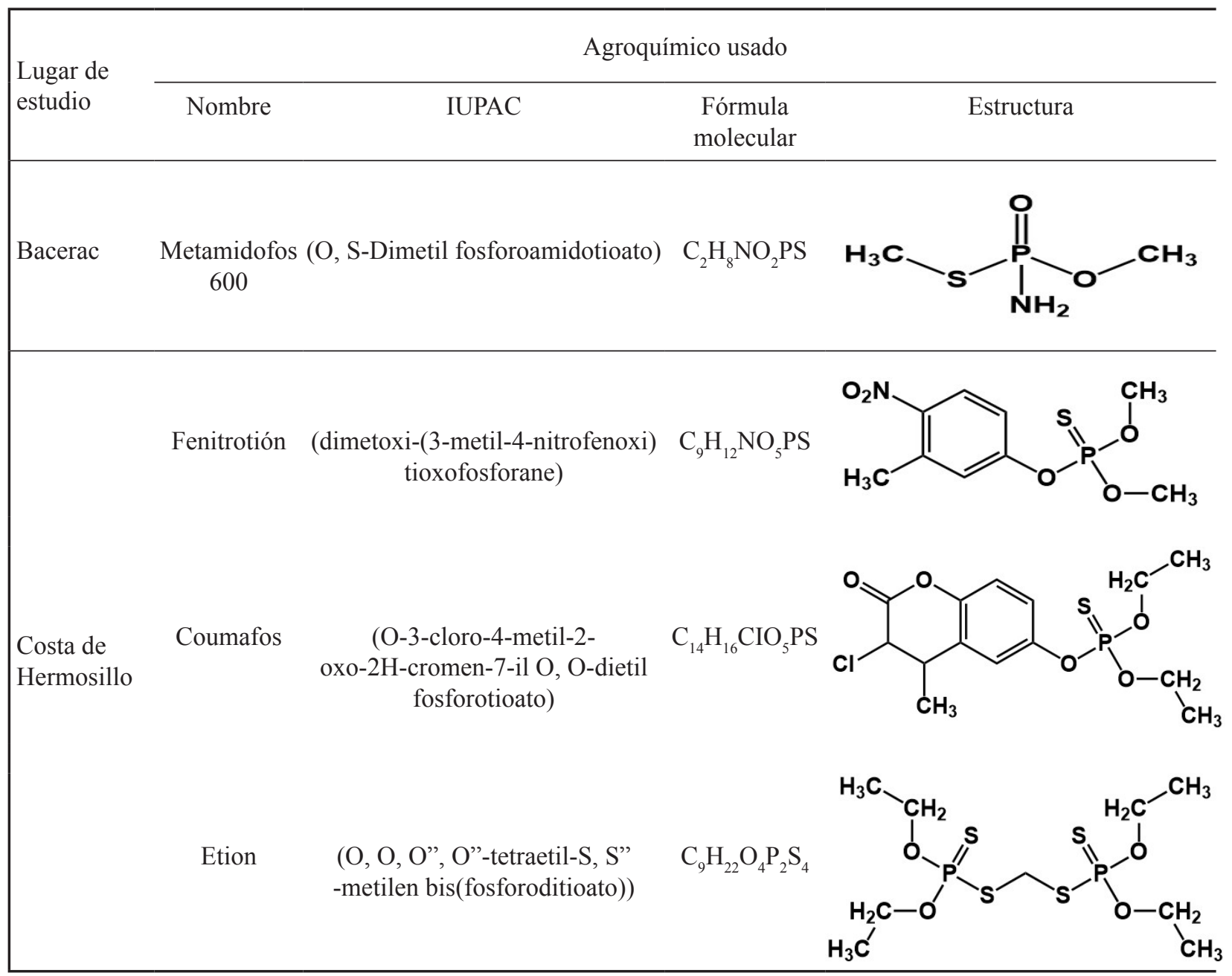

Fuente: elaboración propia con datos tomados de las Fichas de Datos de Seguridad (FDS) (National Center for Biotechnology Information, 2018). 
De los participantes de Bacerac, 92\% inició en el trabajo agrícola siendo menor de 18 años. El promedio de edad de los participantes es de $52 \pm 24$ años y la media de años de trabajar como agricultor es de $40 \pm 23$ años. De los participantes del municipio, $75 \%$ dijo conocer los riesgos en la salud por la aplicación de agroquímicos, pues dicen observar un pictograma de calavera con tibias cruzadas en el envase del producto. No obstante, el mismo porcentaje manifiesta que omite la lectura de las etiquetas y de las instrucciones de aplicación del producto en el cultivo, que se especifican según la NOM-232-SSA1-2009 (DOF-SSA, 2010), además de la información insuficiente en el etiquetado del envase que incluye términos técnicos difíciles de entender incluso para los especialistas (Badii y Varela, 2008).

En el campo de la Costa de Hermosillo se cultiva uva de mesa para exportación. Las mujeres recolectoras que participaron en el estudio tienen una jornada laboral de 8 horas de lunes a sábado. El promedio de edad de las participantes es de $42 \pm 20$ años y tienen 14 años que llegan a laborar al campo anualmente.

En cuanto a la indumentaria de trabajo en Bacerac, 100\% de la muestra indicó qué usa de forma común cuando aplican el producto: zapato cerrado, pantalón de mezclilla, camisa de manga larga y un paño de tela que cubre nariz y boca. En la Costa de Hermosillo, 100\% manifestó que usa zapato cerrado, pantalón largo y camisa de manga larga. En México se cuenta con la Norma Oficial Mexicana NOM-256-SSA1-2012; en ella se establecen las condiciones sanitarias que deben cumplir las personas que apliquen plaguicidas sólo como prestación de servicio (DOF-SSA, 2013). En la normatividad no se incluyen otras actividades en donde haya personal ocupacionalmente expuesto a ese tipo de compuestos de forma residual y prolongada. Por lo anterior, no es claro el compromiso que tiene el Estado y los patrones en relación con el equipo de protección que deben portar los agricultores y los jornaleros a fin de prevenir los efectos a largo plazo que provocan los agroquímicos, ya que una exposición crónica aún en dosis bajas puede deteriorar la salud y el ADN de los trabajadores agrícolas y su descendencia (Bolognesi et al., 2011; Migliore et al., 2011).

Las manifestaciones clínicas referidas por los trabajadores de ambos campos agrícolas se presentan en el cuadro 1. Este cuadro muestra el porcentaje de recurrencia general, con una o más de las sintomatologías. Prevalecen los dolores de cabeza y los mareos, seguidos por problemas de la piel y los respiratorios, síntomas que se dan tanto por las condiciones climáticas del lugar de labor como por la falta de equipo de protección personal (PROCCYT, 2016). En cuanto al daño en el sistema digestivo, 66\% de las personas estudiadas del municipio de Bacerac manifestó molestias, contra 33\% de las mujeres de la Costa de Hermosillo; lo anterior se puede explicar porque las recolectoras realizan la actividad de ingestión de alimentos fuera de la zona agrícola, pues cuentan con un horario de comida de 12:00 a 13:00 h y son transportadas a la zona de residencia.

\section{CUADRO 1}

Manifestaciones clínicas mencionadas por los jornaleros agrícolas en campos de Sonora

\begin{tabular}{|llcc}
\hline Manifestación clínica & $\begin{array}{l}\text { Grado 1 } \\
\text { Intoxicación leve }\end{array}$ & $\begin{array}{c}\text { Bacerac } \\
(\%)\end{array}$ & $\begin{array}{c}\text { Costa de Hermosillo } \\
(\%)\end{array}$ \\
\hline \multirow{2}{*}{ Digestivo } & $\begin{array}{l}\text { Náusea } \\
\text { Vómitos } \\
\text { Respiratorio }\end{array}$ & 62 & 33 \\
& $\begin{array}{l}\text { Diarrea } \\
\text { Dos }\end{array}$ & 42 & 38 \\
Neurológico & Cefalea & & \\
& Mareo & & \\
Vértigo & Somnolencia & 92 & \\
Piel & Irritación & & 42 \\
\hline
\end{tabular}

Fuente: elaboración propia. 
Diversos estudios dan evidencia de los efectos agudos por exposición a agroquímicos, entre los que se dan comúnmente los síntomas de cansancio, debilidad, mareo, cefalea, náusea, vómito e inclusive casos severos que pueden llevar a perder la vida si no se atienden pronto (Blain, 2011), aunado a que $40 \%$ de las intoxicaciones por agroquímicos es de tipo ocupacional y afecta a uno de cada cuatro trabajadores del campo (Medina, 2001). Por lo anterior, el estudio de la salud de los jornaleros y agricultores mexicanos es fundamental para comprender la situación e incidir en las condiciones de riesgo laboral que llegan a alcanzar y afectar a su familia (CamarenaOjinaga et al., 2012).

En el cuadro 2 se presentan los valores de AChE tanto de los individuos de la muestra control como de los trabajadores agrícolas, además se señala el campo en el que laboran, el sexo y la edad de los participantes. La AChE en hombres varió entre 4920 y $10620 \mathrm{UI} / \mathrm{L}$, con un promedio de $7262 \mathrm{UI} / \mathrm{L}$ y una desviación estándar de 1723 UI/L. En mujeres el valor mínimo observado fue de 3210 UI/L y el máximo de 7600 UI/L, con un promedio de $4851 \mathrm{UI} / \mathrm{L}$ y una desviación estándar de $1540 \mathrm{UI} / \mathrm{L}$. Los valores promedio de AChE se encuentran dentro de la normalidad en ambos grupos de trabajadores. Sin embargo, analizando los datos específicos, se encontró sólo en el sexo femenino valores que muestran inhibición de la colinesterasa en cinco mujeres, quienes presentan valores por debajo de las $3930 \mathrm{UI} / \mathrm{L}$, todas ellas mayores de 40 años.

\section{CUADRO 2}

Valor de la acetilcolinesterasa de la población en estudio

\begin{tabular}{|c|c|c|c|c|c|c|c|c|c|c|c|c|c|}
\hline \multicolumn{14}{|c|}{ Agricultores de Bacerac } \\
\hline \multicolumn{14}{|c|}{ Hombres } \\
\hline Edad (años) & 30 & 39 & 41 & 41 & 44 & 45 & 51 & 54 & 59 & 61 & 75 & & 81 \\
\hline $\mathrm{AChE}(\mathrm{UI} / \mathrm{L})$ & 7650 & 10620 & 8520 & 7560 & 5100 & 6210 & 4920 & 6230 & 8100 & 5950 & 6870 & & 9420 \\
\hline \multicolumn{14}{|c|}{ Jornaleras de la Costa de Hermosillo } \\
\hline \multicolumn{14}{|c|}{ Mujeres } \\
\hline Edad (años) & 23 & 29 & 29 & 30 & 34 & 41 & 43 & 48 & 50 & 52 & 57 & 58 & 63 \\
\hline $\mathrm{AChE}(\mathrm{UI} / \mathrm{L})$ & 7600 & 7450 & 4100 & 5900 & 4800 & 3900 & 4250 & 4710 & 3780 & 3210 & 6600 & 3450 & 3320 \\
\hline \multicolumn{14}{|c|}{ Muestra control } \\
\hline \multicolumn{5}{|c|}{ Hombre } & Mujer & & & & & & & & \\
\hline Edad (años) & 18 & 19 & 19 & 18 & 19 & & & & & & & & \\
\hline $\operatorname{AChE}(\mathrm{UI} / \mathrm{L})$ & 9420 & 8900 & 9750 & 10510 & 9600 & & & & & & & & \\
\hline
\end{tabular}

Fuente: elaboración propia.

Un valor bajo de actividad de la colinesterasa en tejidos es evidente signo de que se ha producido algún tipo de exposición a un agente inhibidor de esta enzima; es así que las mujeres poseen mayor riesgo de exposición, lo cual refuerza lo encontrado por Palacios-Nava (2003), quien reporta que el ser hombre es un factor protector en caso de presentarse intoxicación subaguda. Por otra parte, las mujeres muestran factores biológicos inherentes al sexo femenino tales como menor estatura, fragilidad física, mayor proporción de grasa corporal, lo que las pone en desventaja ante los contaminantes (Arellano-García et al., 2012), además de su compromiso reproductivo y la facilidad del cromosoma X para sufrir rupturas en comparación con el resto de los cromosomas (Fenech y Bonassi, 2011). Lo anterior coincide con los informes de la Organización Mundial de la Salud y el Programa de Naciones Unidas para el Medio Ambiente; estas instituciones revelan que el envenenamiento por agroquímicos afecta mayormente a las mujeres (Nivia, 2010). 


\section{Conclusiones}

En los sujetos participantes se encontró un alto porcentaje de valores normales de la actividad de la acetilcolinesterasa. Aunque se dificulta interpretar los resultados al no contar con una línea base de preexposición, éstos se pueden utilizar como indicadores biológicos de exposición a agroquímicos organofosforados y ser parte importante del seguimiento y la detección de los efectos tóxicos producidos. Los resultados son concluyentes: deben intensificarse los esfuerzos para capacitar y actualizar permanentemente al personal técnico profesional, incrementar la toxicovigilancia y fortalecer acciones de prevención y educación hacia la comunidad, ya que también evidencian que la intoxicación por agroquímicos no sólo se da por no usar equipo de protección adecuado sino que también está vinculada con factores culturales y de género.

\section{AnÁLISIS PROSPECTIVO}

Los resultados hallados muestran una afectación mayor en la salud de las mujeres jornaleras de la Costa de Hermosillo que en los agricultores del municipio de Bacerac. Las mujeres representan una parte importante de la fuerza de trabajo agrícola y se encuentran más expuestas a agroquímicos que los hombres. En pláticas con algunas de ellas, manifestaron cumplir con el rol de género y realizar sin ninguna protección el lavado de la ropa que ocupan en las labores de campo, así como la de su pareja sentimental y demás familiares; a esto se agrega las cercanías de las zonas habitacionales a los campos agrícolas. De las mujeres en estudio, más de $50 \%$ se encuentra en etapa reproductiva, a lo que se suma el constante riesgo de toxicidad aguda o crónica con efectos inmunosupresores, hormonales, endócrinos o reproductivos; es una problemática que no ha recibido suficiente atención. Este artículo tiene el objetivo de mostrar el posible daño causado por la exposición a agroquímicos organofosforados, por lo que es urgente que el país cuente con una norma oficial mexicana aplicable en todos los centros de trabajo donde se realicen actividades agrícolas que van desde la preparación del terreno hasta la cosecha, además de que se establezcan las disposiciones que deberán adaptarse a los centros de trabajo a efecto de prevenir los riesgos de los trabajadores que desarrollan actividades agrícolas. Por lo anterior, se deben retroalimentar los resultados encontrados con los grupos estudiados y abrir un canal de interlocución entre gobierno, empresarios, investigadores y trabajadores agrícolas para responder mejor a sus necesidades al estar en riesgo de dañar su salud. Asimismo, es necesario trabajar con un enfoque de atención al cumplimiento de los objetivos de la Agenda 2030 para el Desarrollo Sostenible, la cual está centrada en el objetivo estratégico de propiciar sistemas agrícolas y alimentarios inclusivos y eficientes (FAO, 2016). Por tanto, la agricultura y la alimentación deben estar en un ambiente con equidad de género y que tenga como garantía la salud pública, el desarrollo humano y el cuidado de los recursos naturales aprovechando la experiencia de países que han utilizado alternativas ecológicas para la dependencia de los agroquímicos (Pérez et al., 2010).

\section{REFERENCIAS}

Alavanja, M., Hoppin, J. y Kamel, F. (2004). Health effect of chronic pesticide exposure: cancer and neurotoxicity. Annual Review of Public Health, 25, 155-197.

Albert, L. A. (2015, 17 de abril). Panorama de los plaguicidas en México. La Jornada.

Arellano-Aguilar, O. y Rendón, J. (2016). Greenpeace. Disponible en http://m.greenpeace.org/mexico/Glo$\mathrm{bal} / \mathrm{mexico} /$ Graficos/2016/comida-sana/Plaguicidas_en_agua_ok_EM.pdf. Consultado el 9 de febrero de 2018. 
Arellano-García, M. E., Camarena-Ojinaga, L., Von-Glascoe, Ch., Ruiz-Ruiz, B., Zúñiga-Violante, E. y Montaño-Soto, T. (2012). Daño genotóxico en mujeres y hombres expuestos a plaguicidas en cuatro localidades de Baja California, en Género, ambiente y contaminación por sustancias químicas (pp. 95-113). México: Semarnat.

Badii, M. y Varela, S. (2008). Insecticidas organofosforados: efectos sobre la salud y el ambiente. Culcyt/Toxicología de Insecticidas, 5(28), 5-17.

Blain, P. (2011). US National Library of Medicine National Institutes of Health. Organophosphorus poisoning (acute). Disponible en https://www.ncbi.nlm.nih.gov/pubmed/21575287. Consultado el 16 de febrero de 2018.

Bolognesi, C., Creus, A., Ostrosky-Wegman, P. y Marcos, R. (2011). Micronuclei and pesticide exposure. Mutagenesis, 26(1), 19-26.

Camarena-Ojinaga, L., Von Glascoe, Ch., Arellano-García, M. E., Zúñiga-Violante, E. y Martínez-Valdés, C. (2012). Agroquímicos y mujeres indígenas jornaleras en Baja California, en Género, ambiente y contaminación por sustancias quimicas (pp. 67-68). México: Semarnat.

Centralab. (2016). Centralab. Disponible en http://www.centralab.com.ar/imprimir_impresionTodo.php. Consultado el 17 de febrero 2018.

CICOPLAFEST (Comisión Intersecretarial para el control del Proceso y Uso de Plaguicidas, Fertilizantes y Sustancias Tóxicas). (1991). Secretaría de Agricultura, Ganadería, Desarrollo Rural, Pesca y Alimentación. Disponible en http://biblioteca.semarnat.gob.mx/janium/Documentos/Ciga/agenda/PP03/catalogo.pdf. Consultado el 17 de febrero de 2018.

Cisneros-González, E. (1990). Zona de trabajo, en Estudio de comunidad de los campos de la calle 36 norte Costa de Hermosillo, Sonora (pp. 1-5). Hermosillo: Universidad de Sonora.

Comunica Campeche. (2016, 23 de septiembre). Parten jornaleros campechanos a trabajar a Sonora. Comunica Campeche.com.

DOF-SSA. (2010). Diario Oficial de la Federación, Norma Oficial Mexicana NOM-232-SSA1-2009 Plaguicidas: que establece los requisitos del envase, embalaje y etiquetado de productos grado técnico y para uso agrícola, forestal, pecuario, jardinería. Disponible en http://dof.gob.mx/nota_detalle.php?codigo=5139018\&fecha $=13 / 04 / 2010$. Consultado el 15 de febrero de 2018.

DOF-SSA. (2013). Diario Oficial de la Federación,Norma Oficial Mexicana NOM-256-SSA1-2012. Condiciones sanitarias que deben cumplir los establecimientos y personal dedicados a los servicios urbanos de control de plagas mediante plaguicidas. Disponible en http://dof.gob.mx/nota_detalle.php?codigo=5286029\&fecha $=29 / 01 / 2013$. Consultado el 15 de febrero de 2018 .

Dosemeci, M., Alavanja, M. C., Rowland, A. S., Mage, D., Zahm, S. H., Rothman, N., Lubin, J. H., Hoppin, J. A., Sandler, D. P. y Blair, A. (2002). A quantitative approach for estimating exposure to pesticides in the agricultural health study. Annals of Occupational Hygiene, 46(2), 245-260.

FAO (Food and Agriculture Organization). (2016). La alimentación y la agricultura: claves para la ejecución de la Agenda 2030 para el Desarrollo Sostenible. Disponible en http://www.fao.org/3/a-i5499s.pdf. Consultado el 16 de febrero de 2018.

FAOSTAT (Food and Agriculture Organization Corporate Statical Database) (2017). Food and Agriculture Organization of the United Nations. Disponible en http://www.fao.org/faostat/en/\#data/RFB. Consultado el 9 de febrero de 2018.

Fenech, M. y Bonassi, S. (2011). The effect of age, gender, diet and lifestyle on DNA damage measured using micronucleus frequency in human peripheral blood lumphocytes. Mutagenesis, 26(1), 43-50. 
Gowan Mexicana. (2013). Gowan. Disponible en http://www.gowanmexicana.com/productosgmd.php?producto $=36 \&$ idioma $=3 \&$ categoria $=$. Consultado el 16 de febrero de 2018.

Gutiérrez-Coronado, M. de L., Valenzuela-Quintanar, A. I., Aldana-Madrid, M. de L., Grajeda-Cota, P., Cabrera-Pacheco, R. M., Ballesteros-Vázquez, M. N., Saucedo-Tamayo, M. del S., Ortega-Velez, M. I. y Fierros-Mendiola, D. (2012). Colinesterasa y paraoxonasa séricas como biomarcadores de exposición a plaguicidas en jornaleros agrícolas. Biotecnia, 14(2), 40-46.

INEGI (Instituto Nacional de Estadística y Geografía) (2010). Instituto Nacional de Estadística y Geografía. Disponible en http://www.beta.inegi.org.mx/app/biblioteca/ficha.html ?upc=702825748647. Consultado el 15 de febrero de 2018.

Jiménez-Diaz, M. y Schosinsky-Nevermann, K. (2000). Valores de referencia de colinesterasa plasmática y eritrocitica en población costarricense. Comparación del desempeño clínico de ambas enzimas. Revista Costarricense de Ciencias Médicas, 21(3-4).

Medina, F. (2001). Perfil clínico y epidemiológico en intoxicaciones del Hospital General "Agustin O' Horán" de la SSA, en el estado de Yucatán durante el año 2000. Mérida: Universidad Autónoma de Yucatán.

Migliore, L., Coppede, F., Fenech, M. y Thomas, P. (2011). Association of micronucleus frequency with neurodegenerative diseases. Mutagenesis, 26, 85-92.

Milla-Cotos, O. M. y Palomino-Horna, W. R. (2002). Niveles de colinesterasa sérica en agricultores de la localidad de Carapongo (Perú) y determinación de residuos de plaguicidas inbibidores de la acetilcolinesterasa en frutas y hortalizas cultivadas. Universidad Nacional Mayor de San Marcos.

Mota, S. (2012, 19 de noviembre). México Agropecuario. El economista.

National Center for Biotechnology Information. (2018). PubChem Compound Database Compound CID $=31200,4096,2871$ y 3286. Disponible en https://pubchem.ncbi.nlm.nih.gov/compound/ ethion\#section=Top. Consultado el 17 de febrero de 2018.

Nivia, E. (2010). Las mujeres y los plaguicidas. Disponible en http://www.rapaluruguay.org/publicaciones/e_n_2010.pdf. Consultado el 17 febrero de 2018.

Notimex (2017, 20 de enero). Llegan 60 mil jornaleros a Sonora para trabajar en campos agrícolas. Uniradio noticias.

Palacios-Nava, M. (2003). Aplicación de un instrumento para evaluar exposición a plaguicidas organofosforados, efectos agudos y subagudos en la salud de trabajadores agrícolas. Revista de la Facultad de Medicina $U N A M, 46(1), 22-27$.

Palacios-Nava, M. E. y Moreno-Tetlacuilo, L. (2004). Diferencias en la salud de jornaleras y jornaleros agrícolas migrantes en Sinaloa, México. Salud Pública de México, 46(4), 286-293.

Pérez-Herrera, N. E., Alvarado-Mejía, J. A., Castillo-Burguete, M. T. y González-Navarrete, R. L. (2012). Efectos reproductivos en agricultores expuestos a plaguicidas en Muna, Yucatán, en Género, Ambiente y Contaminación por Sustancias Quimicas (pp. 79-94). México: Semarnat.

Pérez, N., Infante, C., Rosquete, C., Ramos, A., González, C. (2010). Disminuyendo la relevancia de los plaguicidas. Alternativas de su uso. Agroecología, 5, 79-87.

Pose, D., De Ben, S., Delfino, N. y Burger, M. (2000). Intoxicación aguda por organofosforados. Factores de riesgo. Revista Médica de Uruguay, 16, 5-13.

PROCCYT (Protección de Cultivos, Ciencia y Tecnología). (2016). Juntos por una agricultura sustentable. Disponible en http://proccyt.org.mx/pdf/PCT_InformeAnual_pages.pdf. Consultado el 17 de febrero de 2018. 
Rothlein, J., Rohlman, D., Lasarev, M., Phillips, L., Muniz, J. y McCauley, L. (2006). Organophosphate pesticide exposure and neurobehavioral performance in agricultural and nonagricultural hispanic workers. Environmental Health Perspectives, 114(5), 691-696.

Salazar-Adams, A., Moreno-Vázquez, J. L. y Lutz-Ley, A. N. (2012). Agricultura y manejo sustentable del acuífero de la Costa de Hermosillo. Región y Sociedad, 3,155-177.

Seefoó-Lujan, J. (2006). La calidad es nuestra, la intoxicación...jde usted!: atribución de la responsabilidad en las intoxicaciones por plaguicidas agrícolas, Zamora, Michoacán, 1997-2000. Salud Pública de México, $48(5)$.

Segob (Secretaría de Gobernación). (2017). Diario Oficial de la Federación. Disponible en http://www.dof.gob. $\mathrm{mx} /$ nota_detalle.php?codigo $=5472511 \& \mathrm{fecha}=21 / 02 / 2017$. Consultado el 17 de febrero de 2018.

Shelat, A. (2017). MedlinePlus. Disponible en https://medlineplus.gov/spanish/ency/esp_imagepages/9878. htm. Consultado el 13 febrero de 2018.

SIAP (Servicio de Información Agroalimentaria y Pesquera). (2014). Secretaría de Agricultura, Ganadería, Desarrollo Rural, Pesca y Alimentación. Disponible en https://www.gob.mx/cms/uploads/attachment/ file/92738/Cuadros_tabulares_2014.compressed.pdf. Consultado el 16 de febrero de 2018.

SIAP (Servicio de Información Agroalimentaria y Pesquera). (2016). Anuario Estadístico de la Producción Agrícola, México: Gobierno de México.

Silveira, M. I., Amarillas, V. T., Aldana, M. de L. y Zuno, F. (2011). Valoración del riesgo de exposición a insecticidas organofosoforados en adultos del sexo masculino en Sonora, México. Ciencia@UAQ, 4(2), 70-81.

Tecles, F. y Cerón, J. (2003). Determinación espectrofotmétrica de colinesterasa en sangre entera de animales domésticos: factores pre y analíticos. Anales de Veterinaria, 19, 61-76.

Tourliere, M. (2015, 29 de abril). Se usan en México 29 agroquímicos prohibidos en otros países. Proceso.

CC BY-NC-ND 